\title{
NYCTHEMERAL AND MONTHLY OCCUPATION OF THE FISH ASSEMBLAGE ON A SHELTERED BEACH OF BAÍA NORTE, FLORIANÓPOLIS, SANTA CATARINA STATE, BRAZIL
}

\author{
Gisela Costa Ribeiro ${ }^{1}$, Marcelo Soeth ${ }^{2}$, Vinicius Krischnegg Andrade ${ }^{1}$, \\ Henry Louis Spach ${ }^{2}$ and André Pereira Cattani ${ }^{2, *}$ \\ ${ }^{1}$ Universidade Federal de Santa Catarina \\ (Campus Universitário Reitor João David Ferreira Lima, 88040-900 Florianópolis, SC, Brasil) \\ ${ }^{2}$ Universidade Federal do Paraná, Centro de Estudos do Mar \\ (Caixa Postal 50002, 83255-000 Pontal do Paraná, PR, Brasil) \\ *Corresponding author: cattani.andre@gmail.com
}

http://dx.doi.org/10.1590/S1679-87592014065606203

\begin{abstract}
A B S T R A C T
Interpreting fish community records is challenging for several reasons, including the lack of past ichthyofauna data, the cyclical temporal variations in the community, and the methodology employed, which usually underestimates fish assemblages. The objective of this study was to describe short-scale and meso-scale (nycthemeral period and months, respectively) temporal variations in the ichthyofauna composition and structure of a sheltered beach of Baía Norte (Florianópolis, Santa Catarina state, Brazil), using a capéchade net. Samples were collected monthly for a period of 48 hours. During the period from December 2010 to November 2011, a total of 19,302 individuals belonging to 89 species and 39 families were captured. The number of individuals that were sampled during the day and/or night was dependent on the sampling month. On average, the daytime assemblage was more abundant and different in structure and composition than the nighttime assemblage. Of the eight species that had the highest Index of Relative Importance (\%IRI), five had higher variations (ANOVA F) between the day and night than between the months. This finding reinforced the need for sampling during both the day and night. The capéchade net effectively captured demersal and pelagic individuals in a broad range of sizes.
\end{abstract}

\section{RESUMO}

\begin{abstract}
A interpretação dos registros da comunidade ictíica têm se mostrado um desafio por diversos motivos, entre eles a falta de dados pretéritos, existência de variações temporais cíclicas na comunidade e pelos métodos utilizados que geralmente subamostram a assembleia. Neste sentido, o objetivo do presente trabalho foi descrever o padrão de variação temporal (nictemeral e mensal) na composição e na estrutura da ictiofauna de uma praia abrigada da Baía Norte de Florianópolis, usando uma rede capéchade. Coletas foram realizadas mensalmente, integrando um período de 48 horas/mês. Durante os meses de dezembro de 2010 a novembro de 2011, 19302 indivíduos distribuídos em 89 espécies e 39 famílias foram capturados. O número de indivíduos capturados durante o dia e/ou noite foi dependente do mês de coleta. Em média, a assembleia foi mais abundante durante o dia, diferindo em estrutura e composição do período da noite. Das oito espécies com maior Índice de Importância Relativa (IRI\%), cinco apresentaram maior variação (F ANOVA) entre o dia e a noite do que a verificada entre os meses, o que reforça a necessidade de amostragens durante o dia e a noite. A rede capéchade foi eficiente na captura de indivíduos demersais e pelágicos com ampla faixa de tamanho.
\end{abstract}

Descriptors: Coastal zone, Ichthyofauna; Temporal cycles. Descritores: Zona costeira, Ictiofauna, Ciclos temporais.

\section{INTRODUCTION}

Rapid population growth threatens the ecological integrity of coastal environments due to the intense occupation of this narrow region and the overexploitation of nearby fishery resources (HOEFEL, 1998; KENNISH, 2002; FAO, 2010).
Sandy beaches make up a large part of the Brazilian coastal environments (HOEFEL, 1998; BORZONE et al., 2003), and many fish species of ecological and economic interest use these regions to feed, reproduce and develop (RUPLE, 1984; ARAUJO et al., 2008).

In beach environments, the seasonal movements of the ichthyofauna seem to be related to 
the ontogenetic changes within each species (PESSANHA et al., 2003). Species typically stay for short periods of time because this accords with their patterns of recruitment and reproduction, which are synchronized to seasonal abiotic fluctuations (GIBSON et al., 1993; PESSANHA et al., 2003; FÉLIX et al., 2007). In addition, daily fish movement is likely dependent on individual physiological and ecological needs, such as feeding and protection against predators (SUDA et al., 2002; PESSANHA et al., 2003; GAELZER; ZALMON, 2008), which are often affected by physicochemical conditioners, such as turbidity, turbulence, salinity and depth, in the environment (LIN; SHAO, 1999; FÉLIX et al., 2007).

When compared to other environments, beaches provide less shelter for fishes (NAGELKERKEN et al., 2000). However, beach environments usually have high food availability (MCLACHLAN; DORVLO, 2005). Moreover, these areas may have high turbidity and turbulence, which could potentially decrease predation risk for small fishes (ROSS et al., 1987; FÉLIX-HACKRADT, 2010). Fish move into the intertidal zone at night to avoid piscivorous fishes, whereas during the day, they avoid the intertidal zone due to the presence of piscivorous birds (WRIGHT, 1989). The movement of fish species in and out of the beach environment can lead to short-term fluctuations in various community parameters that often exceed the long-term variability (LASIAK, 1984a). Thus, the diel and seasonal compartmentalization of the fish assemblage may be a key factor in understanding how so many species can be supported in a physically simple habitat (VASCONCELLOS et al., 2011).

Several studies in Brazil have looked at the fish communities in beach zones. Most of these studies have dealt with seasonal variations (GIANNINI; PAIVA-FILHO, 1995; GODEFROID et al., 1997; ROSENTAL ZALMON; GAELZER, 2003; ARAUJO et al., 2008; OLIVEIRA-SILVA et al., 2008). However, a few have also dealt with the variations between day and night in the ichthyofauna assemblage (PESSANHA et al., 2003; BARREIROS et al., 2004; GAELZER; ZALMON, 2008; FÉLIX-HACKDART et al., 2010; VASCONCELLOS et al., 2011) and have used small-sized nets for sampling, mainly beach seines.

On the coast of the state of Santa Catarina, the beach ichthyofauna are still largely unknown, with studies being primarily conducted in the Baía da Babitonga (northern Santa Catarina state) (COSTA; SOUZA-CONCEIÇÃO, 2009; VILAR et al., 2011a, 2011b). Despite the importance of the artisanal fishery in the Florianópolis region (MARTINS; PEREZ, 2008; BASTOS; PETRERE Jr., 2010), we found few studies on the ichthyofauna in this area (RIBEIRO et al., 1997, 1999; SOUZA-CONCEIÇÃO et al., 2005;
CARTAGENA et al., 2011). Therefore, the objective of this project was to describe the temporal variation in the composition and structure of the ichthyofauna community at a sheltered beach in Baía Norte (Florianopolis) using a more robust sampling plan than has usually been used in studies of the ichthyofauna at low-energy beaches in the region.

\section{Material and Methods}

\section{Study Area}

Baía Norte is shaped like a long channel, with a $4 \mathrm{~km}$ opening to the Atlantic Ocean to the North and a narrow channel (370 m wide) that is connected to the Baía Sul (Fig. 1). On both the island and the continent, the rivers flow along (across) a short plain of fluviomarine sediments and discharge into small estuaries along an extensive zone of mangrove forests and salt marshes (PAGLIOSA; BARBOSA, 2006). The system is dominated by wind waves and has a low capacity to rework coarse sediment, except in high-energy events, which can give rise to reflective beaches that are associated with muddy floodplains (SILVEIRA et al., 2011). Four high-density municipalities, which comprise a total population of 702,249 (IBGE, 2010), surround Baía Norte.

Índio beach $\left(27^{\circ} 28^{\prime} 33.90\right.$ 's and $48^{\circ} 32^{\prime} 0.75^{\prime \prime} \mathrm{W}$ ) is a wave-sheltered beach (JACKSON et al., 2002) that faces Northwest (Fig. 1). Nearby, there are large fluvial inputs from the Ratones and Veríssimo Rivers, which are approximately $1.7 \mathrm{~km}$ from the sampling site. The tidal regime is semidiurnal, with an average amplitude of $0.52 \mathrm{~m}$ (SORIANO-SIERRA; SIERRA DE LEDO, 1998). The physicochemical variations respond mainly to continental input, and the environment is usually mesotrophic (SIMONASSI et al., 2010). The salinity at this beach is generally high (above thirty), and the temperature fluctuates from 16 to $28^{\circ} \mathrm{C}$ (MACIEL et al., 2010; SIMONASSI et al., 2010).

\section{Data Sampling}

Monthly sampling was conducted from December 2010 to November 2011 during the first neap tide of each month. Samples were collected using a fixed net (capéchade) (for an illustration of the fishing net, refer to Valest et al. (2010)), which was designed to operate at depths of 0.5 to $2.0 \mathrm{~m}$ and is composed of a barrier (20 m long; $2.0 \mathrm{~m}$ high; mesh: $13.0 \mathrm{~mm}$ ) and three concave nets (mesh: $13.0 \mathrm{~mm}$ to $6.0 \mathrm{~mm}$ ). The capéchade or Fyke, is a passive sampler commonly used in shallow estuarine areas and is well adapted to low energy environments and to intertidal habitats (LOUIS et al., 1995; IGNÁCIO; SPACH, 
2009). One major advantage of this sampling gear is that the sample unit area can be large. However, the sample area cannot be defined, and effective sampling depends on tidal flow and fish movement (ROZAS; MINEILO, 1997). The net was set up at the same site at each sampling time, at a depth of 0.5 to $2 \mathrm{~m}$, and it remained there for 48 hours. Fish were harvested in the morning and at night to give a total of two daytime and two nighttime samples, i.e. four monthly samples.

The specimens were weighed $(\mathrm{g})$, measured [total length (TL) and standard length (SL)] and identified following Figueiredo and Menezes (1978, 1980, 2000), Menezes and Figueiredo (1980, 1985), Barletta and Corrêa (1992) and Marceniuk (2005).
In situ" recordings of surface water temperature and salinity were performed every 12 hours (4 recordings/month) using an Incotherm mercury thermometer (precise to $0.1^{\circ} \mathrm{C}$ ) and an RTS/101 ATC portable refractometer, respectively. Water transparency was measured every 24 hours $(2$ recordings/month) using a Secchi (cm) disk. Six sediment samples were collected at the site (three in the swash zone and three parallel to the first two meters of depth) and analyzed using the methods outlined in Suguio (1973). Rainfall and wind data were provided by the EPAGRI/CIRAM meteorological station (Florianopolis) (2734'41.89'S and 48'30'32.79''W), which was $12 \mathrm{~km}$ from the study area.

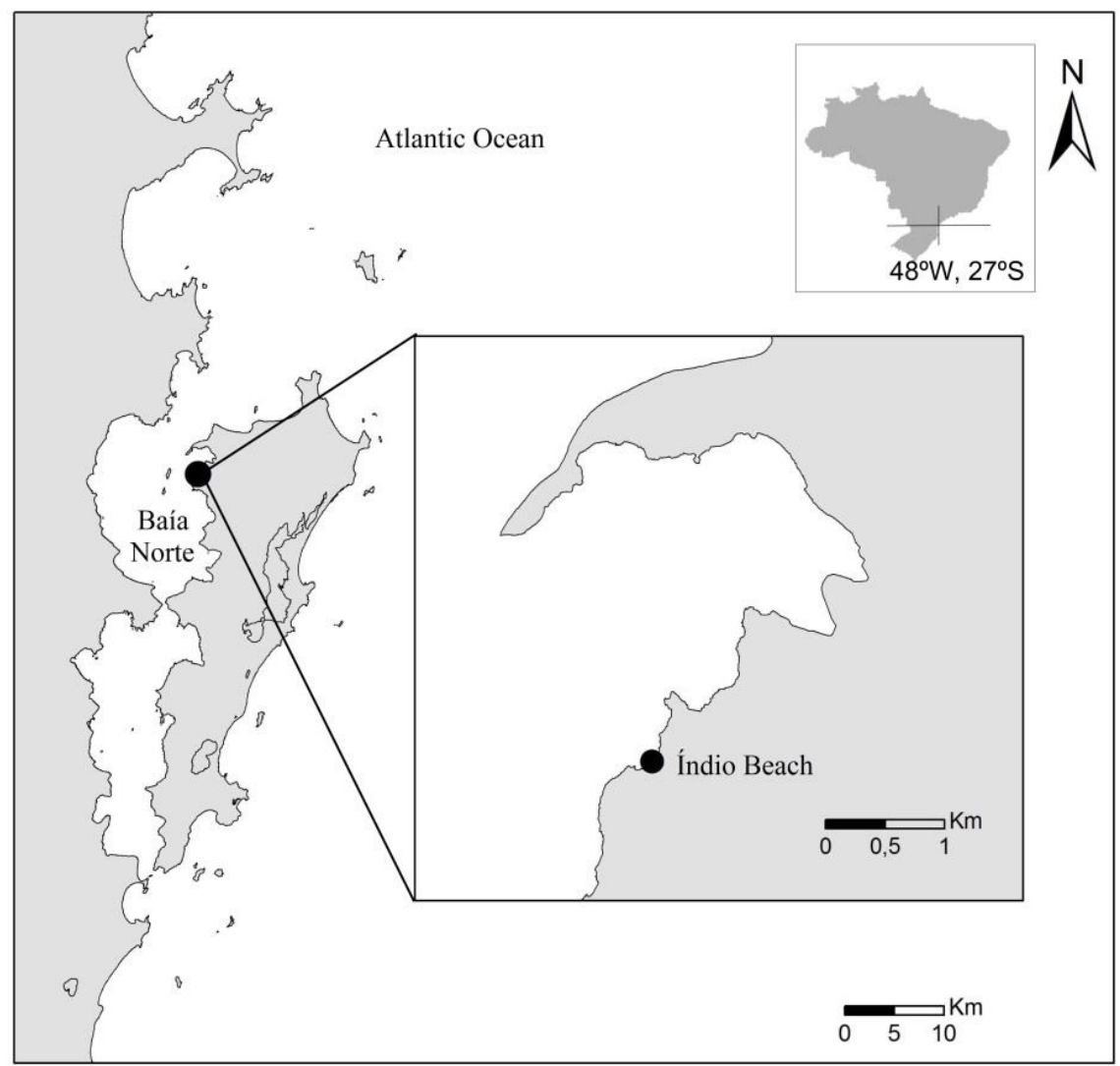

Fig. 1. Map of Brazil showing the location of the North Bay of Florianópolis and Índio Beach $\left(27^{\circ} 28^{\prime} 33.90^{\prime \prime} \mathrm{S}\right.$ and $\left.48^{\circ} 32^{\prime} 0.75^{\prime \prime} \mathrm{W}\right)$. 


\section{Data Analysis}

Species richness was calculated using PRIMER 6.0 (Plymouth Marine Laboratory, England). In addition, the nonmetric multidimensional scaling technique (MDS) from this statistical package was used to study the temporal (month and time of day) variations in the composition and abundance of the species caught over the twelve sampling months. To calculate the MDS, a Bray-Curtis similarity matrix was produced using transformed data $(\log (x+1))$ (CLARKE, 1993). The statistical significance between the groups formed using the MDS were evaluated using an analysis of similarity (ANOSIM) (CLARKE; GORLEY, 2006). Analysis of similarity percentages (SIMPER) were used to identify which species were mainly responsible for the similarities in each group defined by the MDS and for the dissimilarities between the groups (more discriminant species) (CLARKE; WARWICK, 1994).

The most important fish species from the assemblage in each period were obtained using an Index of Relative Importance (\%IRI), which was a modified version of that of Pinkas et al. (1971), and calculated as $[(\% \mathrm{~N}+\% \mathrm{~W}) * \% \mathrm{FO}] * 100$, where $\% \mathrm{~N}=$ percentage of the number of individuals of each species over the total number of individuals; $\% \mathrm{~W}=$ percentage by weight for each species over the total weight; $\% \mathrm{FO}=\mathrm{n} / \mathrm{N} * 100$, where $\mathrm{n}=$ number of samples where the species was present; and $\mathrm{N}=$ total number of samples.

A one-way ANOVA (month as fixed factor) was used to examine the differences between the monthly transparency averages. A two-way ANOVA (month and time of day: day and night as fixed factors) was used to examine the differences between the averages from the day, the night, and the month, as well as differences in the environmental parameters (salinity and temperature), abundance, biomass, and fish species richness. In addition, this model was used to examine the differences between the averages of the abundance and total length of the eight main species (according to \%IRI) from the day, the night, and the months. Before each ANOVA was calculated, the data were tested for homogeneity of variance (Bartlett's test) and normality of the distributions (KolmogorovSmirnov test). To satisfy these ANOVA assumptions, the abundance and biomass data were transformed $[\log (x+1)]$. When significant differences $(p<0.05)$ were found, a Tukey HSD (Honestly Significant Difference) test was used to determine which averages were significantly different. With respect to the total length, only the months in which the species occurred both during the day and at night were included in the analysis. When it was impossible to satisfy ANOVA assumptions, differences were considered significant when $\mathrm{p}<0.01$ (UNDERWOOD, 1997). The relationships among the physicochemical parameters (salinity, transparency, temperature and rainfall), biological parameters, and abundance of the eight main species were verified using a Spearman correlation $(\mathrm{p}<0.05)$.

\section{RESULTS}

\section{Environmental variables}

In shallow beach areas, sediment analysis showed high proportions of sand $(91.55 \%)$, gravel (8.44) and organic matter (1.04) (silt and clay absent). In areas that were $2 \mathrm{~m}$ deep, there was an increase in the concentrations of silt (33.71\%), clay (7.11) and organic matter (5.18), and a decrease in the concentrations of sand (53.66) and gravel (5.17) at about $2 \mathrm{~m}$ deep. From December 2010 to November 2011 the water temperature varied from 16 to $27.5^{\circ} \mathrm{C}$. Variations between the months were significant, with a distinctive seasonal pattern within the region. On average, the highest temperatures occurred during the day with no significant interactions recorded for month or time of day (Fig. 2a; Table 1). Salinity varied from 28 to 36 during the study period, with significant differences between the months, but no significant difference were observed between day and night. No tendency was observed in the salinity values during the sampling months, and the interaction between the month and the time of day was not significant (Fig. 2b; Table 1). Water transparency varied from 0.3 to $1.3 \mathrm{~m}$ during the sampling months. Significant differences between the months were found, with the highest values recorded in December, April, May and June, and the lowest in November, January, February and March (Fig. 2c; Table 1). High rainfall was recorded in August, September, October, January, and February, and low rainfall was recorded in March, April, May, June and July (Fig. 2d).

\section{Ichthyofauna}

During the study period, a total of 19,302 individuals ( 89 species and 39 families) were caught, which accounted for a biomass of 229,066 kg. The average number of individuals caught varied significantly between the months sampled and between day and night (Fig. 3a). In general, there was no consistent number of individuals seen over the course of the month. On average, the number of specimens was greater during the day, with a significant interaction recorded between the time of day and the sampling month (Fig. 3; Table 1). The average biomass caught varied significantly between the months, but there was no significant difference between the day and night samplings (Fig. 3b), and the 
interaction between month and time of day was not significant (Fig. 3b; Table 1). Of the 39 families recorded in this study, the highest species richness were found in Sciaenidae (13 species), Engraulidae (9), Carangidae (8), Gerreidae and Tetraodontidae (5 each), and Ariidae and Paralichthyidae (4 each) (Table 2). Species richness varied significantly between the months; however, the a posteriori Tukey HDS test did not indicate any significant differences between the monthly averages (Fig. 3c; Table 1).
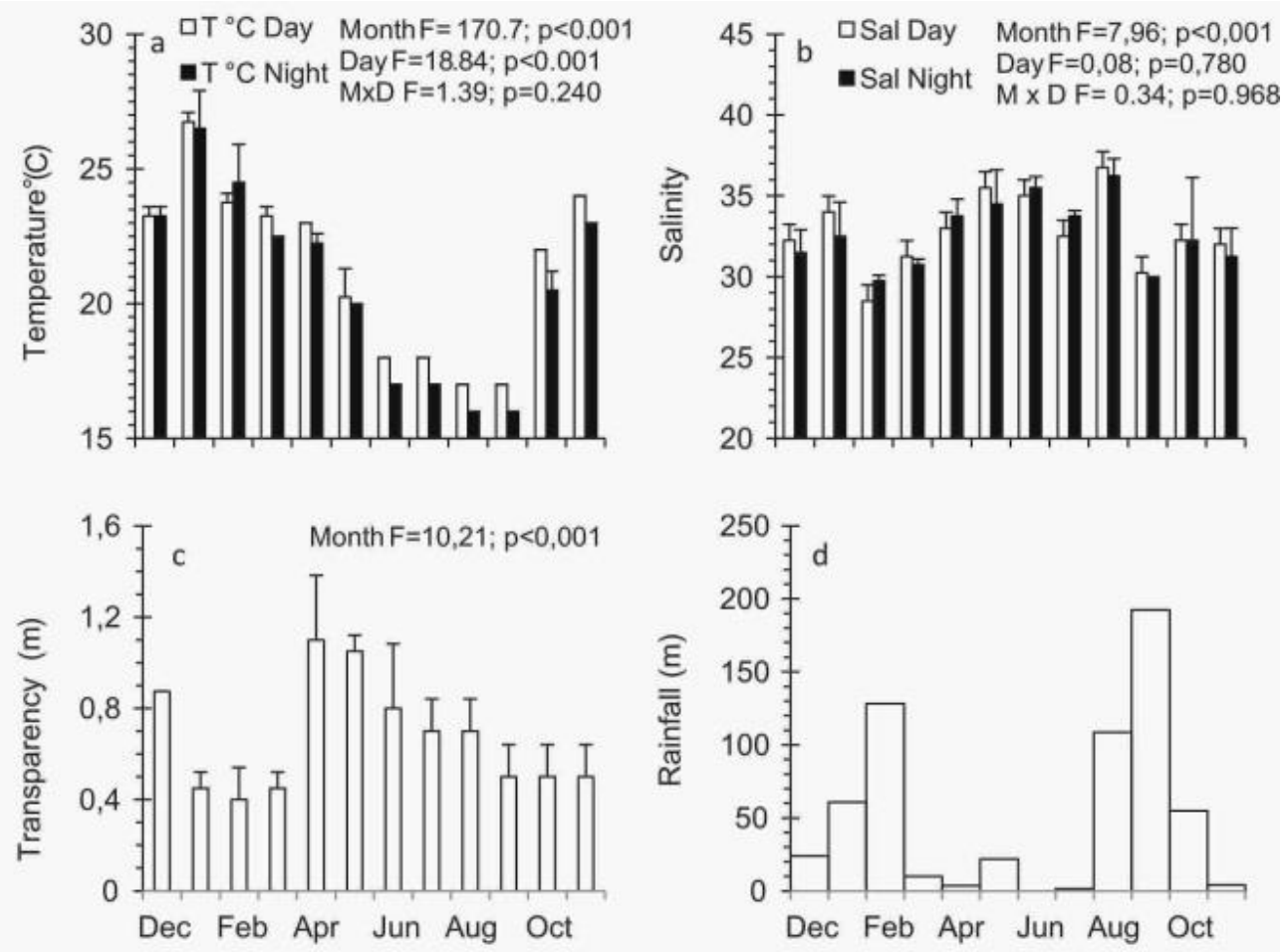

Fig. 2. Monthly average ( \pm standard deviation) of the temperature (a), salinity (b) and transparency (c) for the entire sampling period (day and night) on Índio beach, as well as the rainfall accumulated during the seven days before monthly sampling began (d). Axis x: Month (Dec/2010 to Nov/2011).

Table 1. The results from the Tukey HSD post-hoc test that was used to identify group differences at Índio beach (Florianópolis, months range from December 2010 to November 2011) for: environmental parameters (temperature, salinity and transparency), the number of individuals $(\mathrm{N}(\log (\mathrm{x}+1)))$, biomass $(\log (\mathrm{x}+1))$, and richness $(\mathrm{S}) . \mathrm{D}=$ day; $\mathrm{N}=$ night; $\mathrm{NS}=$ not significant. Averages increase from left to right. $=$ homogeneous groups (Tukey). $* * \mathrm{p}<0.01 ; \mathrm{*}<0.05$.

\begin{tabular}{|c|c|c|c|c|c|c|c|c|c|c|c|c|c|c|c|}
\hline Data set & & & & & & & & & & & & & & Period & Interaction \\
\hline Temperature & $\mathrm{Se}$ & $\mathrm{Au}$ & J & $\mathrm{Jn}$ & $\mathrm{Ma}$ & Oc & Ap & Mar & De & No & $\mathrm{Fe}$ & Ja & ** & $* *$ & NS \\
\hline Salinity & $\mathrm{Fe}$ & $\mathrm{Se}$ & Mar & No & $\mathrm{De}$ & Oc & $\mathrm{Jl}$ & $\mathrm{Ja}$ & Ap & $\mathrm{Ma}$ & Jn & $\mathrm{Au}$ & $* *$ & NS & NS \\
\hline Transparency & $\mathrm{Fe}$ & Mar & Ja & No & Oc & $\mathrm{Se}$ & $\mathrm{Jl}$ & $\mathrm{Au}$ & $\mathrm{Jn}$ & $\mathrm{De}$ & Ma & Ap & ** & & \\
\hline$N((\log (x+1))$ & ال & $\mathrm{Jn}$ & $\mathrm{Au}$ & $\mathrm{Se}$ & $\mathrm{Oc}$ & $\mathrm{Fe}$ & Ap & No & $\mathrm{Ma}$ & $\mathrm{De}$ & Mar & $\mathrm{Ja}$ & $* *$ & $\mathrm{D}>\mathrm{N}^{*}$ & * \\
\hline Biomass ((Log $(x+1))$ & $\mathrm{Jn}$ & Л & Ap & $\mathrm{Se}$ & Mar & $\mathrm{Au}$ & $\mathrm{Ma}$ & $\mathrm{Oc}$ & $\mathrm{Fe}$ & $\mathrm{Ja}$ & No & $\mathrm{De}$ & $* *$ & NS & NS \\
\hline$S$ & Mar & No & $\mathrm{Fe}$ & Oc & $\mathrm{Se}$ & $\mathrm{Au}$ & $\mathrm{Ja}$ & $\mathrm{Jn}$ & $\mathrm{Ma}$ & J & $\mathrm{De}$ & $\mathrm{Ap}$ & * & NS & NS \\
\hline
\end{tabular}



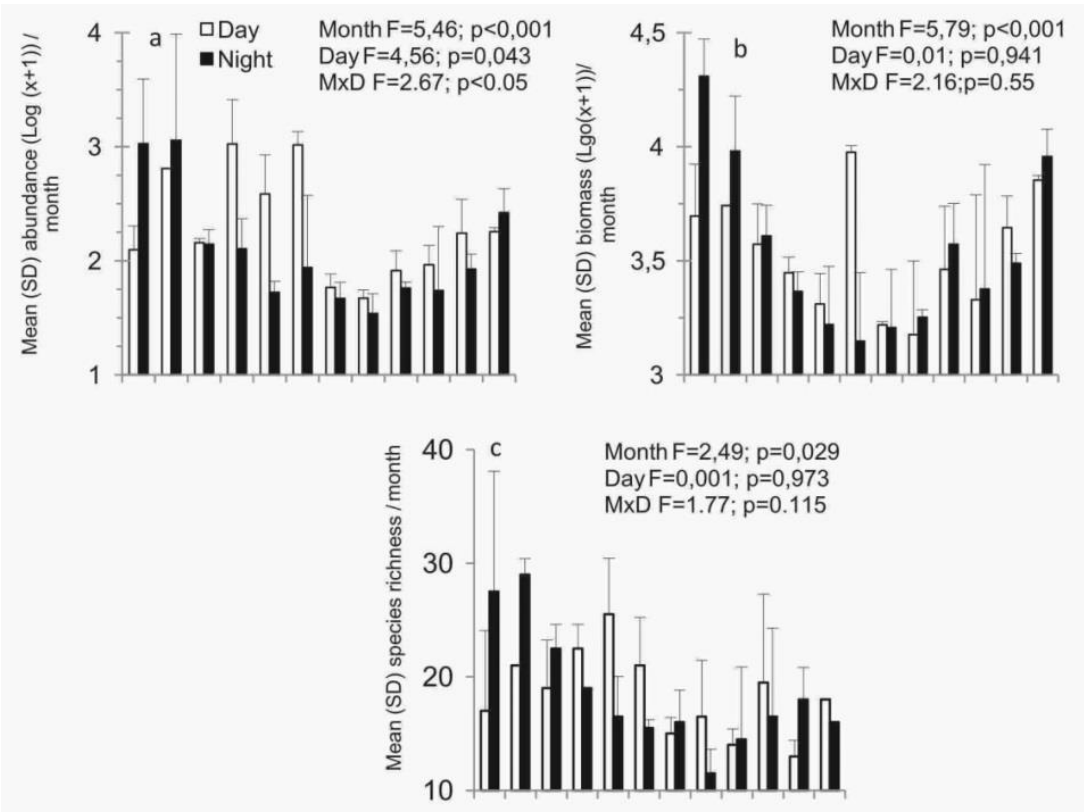

Fig. 3. Monthly average (+ standard deviation) of the (a) number of individuals $(\log (x+1))$, (b) biomass (grams) $(\log (\mathrm{x}+1))$ and $(\mathrm{c})$ species richness for day and night samplings at Índio beach between Dec 2010 and Nov 2011.

Of the species that were caught, 27 were only found at one time of day (day or night). Eleven of them (\% IRI $<0.09$ and $>0.0003$ ) occurred exclusively at night (Ophichthus gomesii, Diplodus argenteus, Centropomus parallelus, Odontesthes argentinensis, Bairdiella ronchus, Anisotremus surinamensis, Pogonias cromis, Porichthys porosissimus, Prionotu snudigula, Macrodon ancylodon and Hemicaran amblyrhynchus). Sixteen (\%IRI $<0.285$ and $>0.001)$ of the species occurred exclusively during the day (Lycengraulis grossidens, Mugil sp., Oligoplites saliens, Trachinotus falcatus, Sphyraena guachancho, Strongylura marina, Peprilus paru, Oligoplites palometa, Scomberomorus brasiliensis, Anchoa marinii, Paralichthys patagonicus, Pomatomus saltatrix, Elops saurus, Synodus foetens, Gobionellus oceanicus and Astroscopus ygraecum (Table 2).

Eight species comprised $85 \%$ of the Index of Relative Importance (\%IRI) (averages for day and night). In order of descending importance (\%IRI for day and night, respectively), they were: Sphoeroides testudineus (29.82 and 6.55), Genidens barbus (7.27 and 26.07), Harengula clupeola (9.52 and 18.63), Anchoviella lepidentostole (18.40 and 5.98), Genidens genidens (3.09 and 13.53), Stellifer rastrifer (5.56 and 6.93), Cetengraulis edentulus (1.38 and 9.89), and Sphoeroides greeleyi (7.09 and 0.88) (Table 2). The analysis of variance showed that of the eight species with the highest \%IRI, S. testudineus, $S$. greeleyi, $G$. genidens, G. barbus and A. lepidentostole presented higher variation in abundance (ANOVA F) between day and night than that which was verified between the monthly samplings (Table 3). Sphoeroides testudines, S. greeleyi and A. lepidentostole were significantly more abundant during the day, and $G$. barbus, G. genidens and S. rastrifer were significantly more abundant at night (Table 3).

MDS, which was based on the abundance of each species, separated the day and night catches reasonably well (Fig. 4). The differences between the day and the night ichthyofauna assemblages were verified using an ANOSIM (Global $\mathrm{R}=0.311$; $\mathrm{p}<0.01$ ), which indicated that the means of separation for the groups were weak. SIMPER analysis recorded a dissimilarity of $67.42 \%$ between the day and the night (Table 4), with the highest contribution from $A$. lepidentostole and S. testudineus during the day, and G. genidens and G. barbus at night (Table 4). MDS showed a clearer separation of the months when compared to the time of day (Fig. 4) and formed 3 groups: G1 (November, December, January and February), G2 (March, April and May), and G3 (June, July, August, September and October). The groups were verified using an ANOSIM, and significant differences were found (Global $\mathrm{R}=0.435 ; \mathrm{p}<0.01$ ). The groups overlapped but were reasonably different from each other. Pairwise comparisons showed that the difference between groups $\mathrm{G} 1 \mathrm{xG} 2$ was greatest (Global $\mathrm{R}=0.562 ; \mathrm{p}<0.01$ ), followed by $\mathrm{G} 1 \mathrm{xG} 3$ (Global $\mathrm{R}=0.426 ; \mathrm{p}<0.01$ ) and $\mathrm{G} 2 \mathrm{xG} 3$ (Global $\mathrm{R}=$ $0.463 ; \mathrm{p}<0.01)$. 
Table 2. The abundance, total biomass, Index of Relative Importance (\%IRI), average total length (TL) ( \pm standard deviation) and range of TL for the species captured during the day and night from December 2010 to November 2011 at Índio beach.

\begin{tabular}{|c|c|c|c|}
\hline & & & \\
\hline Family & Species & $\mathrm{n}$ & $\begin{array}{l}\text { IRI } \\
(\%)\end{array}$ \\
\hline Achiridae & Achinas lineatus & 2 & 0,006 \\
\hline Ariidae & Cathoropss spixii & 58 & 0,409 \\
\hline & Genidens barbus & 252 & 7.273 \\
\hline & Genidens genidens & 92 & 3,098 \\
\hline & Genidens machadoi & 2 & 0,006 \\
\hline Atherinopsidae & Atherinella brasiliensis & 29 & 0,246 \\
\hline & Odontesches argentinentis & & \\
\hline Batrachoididae & Porichthys porosissimus & & \\
\hline Belonidae & Strongylura marina & 2 & 0.007 \\
\hline Carangidae & Chloroscombris chrysunus & 42 & 0,496 \\
\hline & Hemicaranx amblyrhnchus & & \\
\hline & Otigoplites palometa & 4 & 0,005 \\
\hline & Oligoplites saliens & 11 & 0,034 \\
\hline & Otigoplites saurus & 31 & 0.342 \\
\hline & Selene vomer & 64 & 1,044 \\
\hline & Thachinotus carolinus & 14 & 0,065 \\
\hline & Trachinotus falcants & 3 & 0,023 \\
\hline Centropomidae & Centropomus parallelus & & \\
\hline Clupeidae & Harengula clupeola & 1776 & 9,525 \\
\hline & Opisthonema oglinum & 40 & 0,079 \\
\hline & Sandinella brasiliensis & 271 & 1,015 \\
\hline Cynoglossidae & Symphurus tessellatus & 2 & 0,002 \\
\hline Dactylopteridae & Dactyloptenes volitans & 9 & 0,038 \\
\hline Diodontidae & Chilomyctenes spinosus & 3 & 0,048 \\
\hline Elopidae & Elops saunus & 1 & 0,001 \\
\hline Engraulidae & Anchovia chpeoides & 2 & 0,001 \\
\hline & Anchoa januaria & 123 & 0,339 \\
\hline & Anchoviella lepidentostole & 3380 & 18,403 \\
\hline & Anchoa marinii & 3 & 0,004 \\
\hline & Anchoa spinifer & 8 & 0,006 \\
\hline & Anchoa tricolor & 35 & 0,031 \\
\hline & Cetengraulis edentulus & 151 & 1.384 \\
\hline & Engraulis anchoita & 31 & 0,027 \\
\hline & Lycengrualis grossidens & 31 & 0.284 \\
\hline Ephippidae & Chaetodiptens faber & 7 & 0,045 \\
\hline Gerreidae & Diaptenus auratus & 3 & 0.017 \\
\hline & Diapterus rhombeus & 16 & 0,346 \\
\hline & Eucinostomus argenteus & 9 & 0,058 \\
\hline & Eucinostomus gula & 17 & 0,137 \\
\hline & Eucinostomus melanoptenas & 46 & 1,114 \\
\hline Gobildae & Gobionellus oceanicus & 1 & 0.001 \\
\hline Haemulidae & Anisotremus surinamensis & & \\
\hline & Orthoppistis ruber & 10 & 0,225 \\
\hline & Pomadasys corvinaeformis & 6 & 0,015 \\
\hline Hemiramphidae & Hyporhamphus unifasciahus & 3 & 0,014 \\
\hline Lutjanidae & Lutjanus analis & 2 & 0,023 \\
\hline Monacanthidae & Stephanolepix hispidus & 1 & 0,001 \\
\hline Mugilidae & Mugil carema & 605 & 6.06 \\
\hline & Mugil liza & 2 & 0,018 \\
\hline & Mugil sp & 47 & 0,079 \\
\hline Muraenidae & Gymnothorax acellatus & 1 & 0,005 \\
\hline Ophichthidae & Ophichthus gomesii & & \\
\hline Paralichthyidae & Citharichlitys macrops & 40 & 0,358 \\
\hline & Citharichtlyss spilopterus & 78 & 1,551 \\
\hline & Empopus crossotas & 7 & 0,022 \\
\hline & Paralichthys patagonicus & 1 & 0,003 \\
\hline Polynemidae & Polydactylus virginicus & 7 & 0,129 \\
\hline Pomatomidae & Pomatomus saltatrix & 1 & 0,002 \\
\hline Priacanthidae & Heteropriacanthus cruentatus & & \\
\hline Pristigasteridace & Pellona harrower $i$ & 1 & 0,001 \\
\hline Sciaenidae & Baintiella rhonchus & & \\
\hline & Conoscion jamaicensis & 2 & 0,003 \\
\hline & Cymoscion microlepidonus & 6 & 0,007 \\
\hline & Isopisthus paripipinnis & 8 & 0,049 \\
\hline & Larimus breviceps & 7 & 0,023 \\
\hline & Menticirmus americanus & 1 & 0,002 \\
\hline & Macrodon ancylodon & & \\
\hline & Micropogonias furnieri & 32 & 0,769 \\
\hline & Menticirmus tithoralis & 5 & 0,054 \\
\hline & Paralonchurus brasiliensis & 1 & 0,004 \\
\hline & Pogonias cromis & & \\
\hline & Stellifer nastrffer & 176 & 5.561 \\
\hline & Stellifer stellifer & 1 & 0,004 \\
\hline Scombridae & Scomberomonus brasiliensis & 2 & 0,005 \\
\hline Serranidae & Diplectrum radiale & 8 & 0,05 \\
\hline Sparidae & Archosargess rhamboidalis & u & 0,494 \\
\hline & Diplodus argenteus & & \\
\hline Sphyraenidae & Sphyruena guachancho & 8 & 0,02 \\
\hline Stromateidae & Pepritus paru & 1 & 0.005 \\
\hline Synodontidae & Symodus foetens & 1 & 0,001 \\
\hline Tetraodontidae & Lagocephatus laevigatus & 1 & 0,002 \\
\hline & Sphoeroides greeleyi & 379 & 7,099 \\
\hline & Sphoeroider spengleri & III & 1,474 \\
\hline & Sphoeroides testudineus & 547 & 29,829 \\
\hline & Sphoeroides yleri & 9 & 0,019 \\
\hline Trichiuridae & Trichiuras lepturus & 5 & 0,027 \\
\hline Triglidae & Prionotus nudigula & & \\
\hline & Prionotass punctatus & 5 & 0,036 \\
\hline Uranoscopidae & Astrascopus $Y_{\text {graecum }}$ & 1 & 0 \\
\hline & & & \\
\hline & Number of individuals (n) & & \\
\hline & Biomass (g) & & \\
\hline & Number of species & & \\
\hline
\end{tabular}




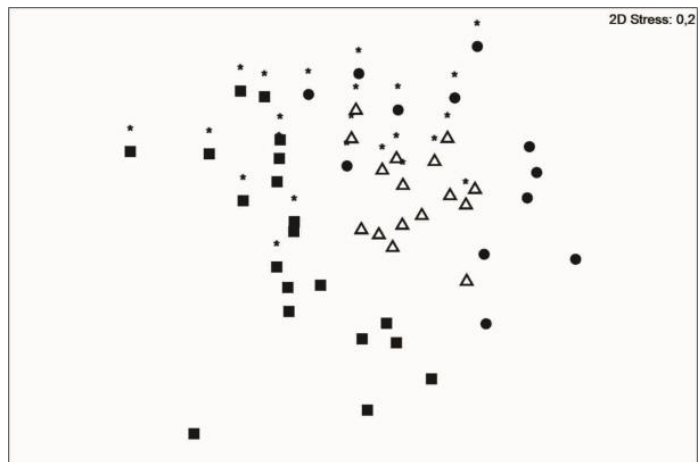

Fig. 4. MDS based on the Bray-Curtis similarity matrix that considered groups G1 (November, December, January and February, triangles), G2 (March, April and May, circles), and G3 (June, July August, September and October, square) and time of day. Nighttime samples are marked with an *. Índio beach, December 2010 to November 2011

The dissimilarity average from the SIMPER analysis for G1xG2 was $62.46 \%$, with G.barbus, $S$. testudineus and $C$. edentulus in G1 and $A$. lepidentostole, G. barbus and $H$. clupeola in G2 contributing the most to the dissimilarity. In the G1xG3 comparison, the average dissimilarity was $66.70 \%$, with $G$. barbus, $S$. testudineus and $C$. edentulus in G1 and G. genidens, S. testudineus and $S$. rastrifer in G3 having the greatest average abundances. The greatest dissimilarity was found between G2xG3 (average of $72.30 \%$ ), with $A$. lepidentostole, G. barbus and H. clupeola in G2 and G. genidens, S. testudineus and S. rastrifer in G3 having the greatest average abundances (Table 4$)$.

A significant positive correlation $(\mathrm{p}<0.05)$ between salinity with transparency, and a significant negative correlation $(p<0.05)$ for salinity with temperature and rain, as well as between rain with transparency were found when environmental data were compared using a Spearman correlation (Table $5)$. When biotic and abiotic data were compared, a significant positive correlation $(\mathrm{p}<0.05)$ was observed for temperature with total biomass, number of individuals, and with the abundance of S. testudineus, G. barbus, H. clupeola, C.edentulus, S. greeleyi and A. lepidentostole. Transparency had a significant negative correlation $(\mathrm{p}<0.05)$ to $G$. barbus and $G$. genidens, and a significant negative correlation $(\mathrm{p}<0.05)$ was also recorded between $G$. barbus and salinity. There was a positive $(\mathrm{p}<0.05)$ and significant correlation for meteorological precipitation with total biomass and $G$. genidens abundance.

Table 3. The results of the Tukey HSD post-hoc test identifying differences at Índio beach (Florianópolis, months range from December 2010 to November 2011) for: abundance (N) and total length (TL) (fixed factors: month and time of day). $\mathrm{D}=$ day; $\mathrm{N}=$ night; $\mathrm{NS}=$ not significant. Average values increase from left to right. $=$ homogeneous groups $(\mathrm{T}=$ Tukey $) . \mathrm{F}=\mathrm{F}$ ANOVA. **p<0.01; *p<0.05. Spte $=$ S. testudineus; Geba $=$ G. barbus; Hacl $=\bar{H}$. clupeola $;$ Anle $=$ A. lepidentostole $;$ Gege $=$ G. genidens; Stra= S. rastrifer; Ceed $=$ C. edentulus; Spgr $=$ S. greeleyi .

\begin{tabular}{|c|c|c|c|c|c|c|c|c|c|c|c|c|c|c|c|c|c|}
\hline$S p$ & \multicolumn{2}{|c|}{ Data } & \multicolumn{13}{|c|}{ Month } & \multirow{2}{*}{$\begin{array}{c}\text { Period } \\
45.29\end{array}$} & \multirow{2}{*}{$\begin{array}{c}\text { Interaction } \\
1.61\end{array}$} \\
\hline \multirow[t]{4}{*}{ Spte } & $\mathrm{N}^{\text {set }}$ & F & & & & & & & 8.39 & & & & & & & & \\
\hline & & $\mathrm{T}$ & JI & $\mathrm{Se}$ & $\mathrm{Ma}$ & $\mathrm{Au}$ & $\mathrm{Ap}$ & Mar & Jn & $\mathrm{Fe}$ & No & $\mathrm{Oc}$ & $\mathrm{Ja}$ & $\mathrm{De}$ & $* *$ & $\mathrm{D}>\mathrm{N}^{* *}$ & NS \\
\hline & TL & F & & & & & & & 2.25 & & & & & & & 1.54 & 1.63 \\
\hline & & $\mathrm{T}$ & $\mathrm{Ja}$ & $\mathrm{Se}$ & $\mathrm{Ap}$ & Mar & $\mathrm{Fe}$ & $\mathrm{Oc}$ & No & Jn & $\mathrm{Au}$ & $\mathrm{De}$ & $\mathrm{Ma}$ & $\mathrm{n}$ & ** & NS & NS \\
\hline \multirow[t]{4}{*}{ Geba } & $\mathrm{N}$ & F & & & & & & & 0.32 & & & & & & & 24.42 & 0.96 \\
\hline & & $\mathrm{T}$ & $\mathrm{Au}$ & $\mathrm{Jn}$ & $\mathrm{Jl}$ & $\mathrm{Se}$ & $\mathrm{Oc}$ & $\mathrm{Ma}$ & Ap & $\mathrm{Fe}$ & Mar & No & $\mathrm{Ja}$ & $\mathrm{De}$ & $* *$ & $\mathrm{D}<\mathrm{N} * *$ & NS \\
\hline & TL & F & & & & & & & 3.55 & & & & & & & 5.08 & 2.48 \\
\hline & & $\mathrm{T}$ & Mar & $\mathrm{Ma}$ & Ap & $\mathrm{Se}$ & No & $\pi$ & De & $\mathrm{Ja}$ & $\mathrm{Fe}$ & Jn & $\mathrm{Oc}$ & $\mathrm{Au}$ & ** & NS & ** \\
\hline \multirow[t]{4}{*}{$\mathrm{Hacl}$} & $\mathrm{N}$ & F & & & & & & & 11.92 & & & & & & & 2.12 & 1.49 \\
\hline & & $\mathrm{T}$ & $\mathrm{Ap}$ & $\mathrm{Au}$ & Лl & No & $\mathrm{Se}$ & $\mathrm{Jn}$ & $\mathrm{Fe}$ & $\mathrm{Ou}$ & $\mathrm{De}$ & Mar & $\mathrm{Ma}$ & $\mathrm{Ja}$ & ** & Ns & NS \\
\hline & $\mathrm{TL}$ & F & & & & & & & 0.03 & & & & & & & 24.21 & 24.33 \\
\hline & & $\mathrm{T}$ & $\mathrm{Ja}$ & Mar & $\mathrm{De}$ & $\mathrm{Ma}$ & $\mathrm{Ou}$ & & & & & & & & & NS & $* *$ \\
\hline \multirow[t]{4}{*}{ Anle } & $\mathrm{N}$ & F & & & & & & & 4.35 & & & & & & & 9.43 & 2.33 \\
\hline & & $\mathrm{T}$ & $\mathrm{Se}$ & No & $\mathrm{Jl}$ & $\mathrm{Ja}$ & $\mathrm{Oc}$ & $\mathrm{Jn}$ & $\mathrm{Fe}$ & De & $\mathrm{Au}$ & $\mathrm{Ap}$ & $\mathrm{Ma}$ & Mar & ** & $\mathrm{D}>\mathrm{N}^{*}$ & NS \\
\hline & TL & F & & & & & & & 8.19 & & & & & & & 1.78 & 1.56 \\
\hline & & $\mathrm{T}$ & Mar & Ap & $\mathrm{Fe}$ & $\mathrm{Ma}$ & $\mathrm{De}$ & $* *$ & & & & & & & & NS & NS \\
\hline \multirow[t]{4}{*}{ Gege } & $\mathrm{N}$ & F & & & & & & & 1.3 & & & & & & & 33.6 & $\begin{array}{c}\text { NS } \\
0.58\end{array}$ \\
\hline & & $\mathrm{T}$ & & & & & & & NS & & & & & & & $\mathrm{D}>\mathrm{N} * *$ & NS \\
\hline & TL & F & & & & & & & 4.92 & & & & & & & 3.26 & 2.18 \\
\hline & & $\mathrm{T}$ & $\mathrm{Ja}$ & $\mathrm{Fe}$ & Mar & No & $\mathrm{De}$ & Jn & $\mathrm{Se}$ & $\mathrm{Oc}$ & $\mathrm{Ap}$ & $\mathrm{Au}$ & ת & & & NS & NS \\
\hline \multirow[t]{4}{*}{ Stra } & $\mathrm{N}$ & F & & & & & & & 2.07 & & & & & & & 5.63 & 0.83 \\
\hline & & $\mathrm{T}$ & Mar & $\mathrm{Ma}$ & $\mathrm{Ap}$ & $\mathrm{Au}$ & $\mathrm{Fe}$ & Jn & $\pi$ & $\mathrm{Ja}$ & $\mathrm{Se}$ & $\mathrm{Oc}$ & No & $\mathrm{De}$ & $* *$ & $\mathrm{D}<\mathrm{N} *$ & NS \\
\hline & TL & F & & & & & & & 11.86 & & & & & & & 0.08 & 0.9 \\
\hline & & $\mathrm{T}$ & $\mathrm{Ja}$ & $\mathrm{De}$ & No & $\mathrm{Oc}$ & $\mathrm{Au}$ & $\mathrm{Ap}$ & $\mathrm{Jn}$ & $\mathrm{Jl}$ & $\mathrm{Se}$ & $* *$ & & & & NS & NS \\
\hline \multirow[t]{4}{*}{ Ceed } & $\mathrm{N}$ & F & & & & & & & 2.49 & & & & & & & 2.05 & 0.48 \\
\hline & & $\mathrm{T}$ & $\mathrm{Se}$ & ת & $\mathrm{Au}$ & $\mathrm{Jn}$ & $\mathrm{Ap}$ & Mar & $\mathrm{Fe}$ & $\mathrm{De}$ & $\mathrm{Ma}$ & No & $\mathrm{Ja}$ & $* *$ & & NS & NS \\
\hline & TL & F & & & & & & & 08.49 & & & & & & & 32.48 & 7.31 \\
\hline & & $\mathrm{T}$ & $\mathrm{Ja}$ & $\mathrm{De}$ & No & $\mathrm{Oc}$ & $\mathrm{Au}$ & $\mathrm{Ap}$ & $\pi$ & $\mathrm{Se}$ & $* *$ & & & & & $\mathrm{D}<\mathrm{N} *$ & ** \\
\hline \multirow[t]{4}{*}{ Spgr } & $\mathrm{N}$ & F & & & & & & & 8.65 & & & & & & & 38.09 & 3.72 \\
\hline & & $\mathrm{T}$ & $\mathrm{Jl}$ & $\mathrm{Se}$ & Jn & $\mathrm{Ma}$ & $\mathrm{Au}$ & $\mathrm{Ap}$ & $\mathrm{Oc}$ & $\mathrm{Fe}$ & No & Mar & $\mathrm{De}$ & Ja & \multirow[t]{3}{*}{ ** } & $\mathrm{D}>\mathrm{N} * *$ & $* *$ \\
\hline & $\mathrm{TL}$ & F & & & & & & & 6.65 & & & & & & & 5.73 & 5.72 \\
\hline & & $\mathrm{T}$ & $\mathrm{Jl}$ & $\mathrm{Ma}$ & $\mathrm{Ap}$ & $\mathrm{Fe}$ & $\mathrm{Au}$ & Mar & No & $\mathrm{Ja}$ & $\mathrm{De}$ & ** & & & & NS & ** \\
\hline
\end{tabular}


Table 4. The analysis of similarity percentages (SIMPER) between the groups formed using MDS. The results show the dissimilarity (\%) of the nine species that contributed the most to each analysis. D = day; $\mathrm{N}=$ night; G1 = November, December, January and February; G2 = March, April and May; G3 = June, July, August, September and October.

\begin{tabular}{lcccc}
\hline Species & $\mathrm{NxD}$ & $\mathrm{G} 1 \mathrm{xG} 3$ & $\mathrm{G} 1 \mathrm{xG} 3$ & $\mathrm{G} 2 \mathrm{xG} 3$ \\
\hline A. lepidentostole & 5.47 & 7.94 & & 9.18 \\
G. genidens & 4.69 & & & \\
S. testudineus & 4,68 & 3,97 & 4,64 & \\
H. clupeola & 4.51 & 5.96 & & 5.11 \\
G. barbus & 4.50 & & 6.23 & 4.03 \\
S. greeleyi & & 4.57 & 4,99 & \\
C. edentulus & & 4.82 & 6.23 & 4.10 \\
S. rastrifer & & & & \\
M. curema & & & & 3.92 \\
\hline
\end{tabular}

Table 5. Spearman correlation for the environmental parameters, total abundance, total biomass, and abundance of the eight main species caught according to the \%IRI. Significant correlations $(\mathrm{p}<0.05)$ are in bold.

\begin{tabular}{lccccc} 
& Temperature & Salinity & Transparency & Rainfall & Abundance \\
\hline Salinity & $\mathbf{- 0 , 3 5 6}$ & & & & \\
Transparency & $-0,340$ & $\mathbf{0 , 5 5 6}$ & & & \\
Rainfall & 0,061 & $\mathbf{- 0 , 3 4 0}$ & $\mathbf{- 0 , 4 3 7}$ & & \\
Abundance & $\mathbf{0 . 6 5 6}$ & $-0,149$ & 0,034 & 0,176 & \\
Biomass & $\mathbf{0 , 5 1 5}$ & $-0,168$ & $-0,076$ & $\mathbf{0 , 3 1 5}$ & $\mathbf{0 . 7 2 3}$ \\
S. testudineus & $\mathbf{0 , 5 5 3}$ & $-0,124$ & $-0,324$ & 0,192 & $\mathbf{0 . 4 8 5}$ \\
G. barbus & $\mathbf{0 , 6 8 8}$ & $\mathbf{- 0 , 4 0 2}$ & $\mathbf{- 0 , 4 9 8}$ & 0,065 & $\mathbf{0 . 4 5 9}$ \\
H. clupeola & $\mathbf{0 , 3 6 9}$ & 0,126 & 0,028 & 0,149 & $\mathbf{0 . 5 4 9}$ \\
A. lepidentostole & 0,271 & $-0,073$ & 0,207 & $-0,135$ & $\mathbf{0 . 4 6 5}$ \\
G. genidens & $-0,060$ & $-0,278$ & $\mathbf{- 0 , 6 3 5}$ & $\mathbf{0 , 3 0 4}$ & -0.028 \\
S. rastrifer & 0,141 & $-0,223$ & $-0,159$ & 0,022 & 0.040 \\
C. edentulus & $\mathbf{0 , 6 1 6}$ & 0,057 & $-0,007$ & $-0,024$ & $\mathbf{0 , 4 4 7}$ \\
S. greelevi & $\mathbf{0 , 6 6 1}$ & $-0,180$ & $-0,347$ & 0,152 & $\mathbf{0 . 5 9 5}$
\end{tabular}

\section{DisCUSSION}

Sediment analysis confirmed the environmental dynamics for a low-energy beach typically sheltered from waves. The deposition of silt, clay, and organic matter at shallow depths was recorded, as was their absence in a short swash zone that had high concentrations of sand due to the action of small waves and/or past high-energy events (JACKSON et al., 2002; SILVEIRA et al., 2011). The sedimentary characteristics found in this study are also commonly found on some estuarine beaches inside Baía de Paranaguá, with coarse sand on the beach face and an associated muddy plain (ROSA; BORZONE, 2008), which are characteristics that generally increase the richness and abundance of the macrofauna (MCLACHLAN; DORVLO, 2005). This type of environment could, theoretically, increase the availability of food at Índio beach.

Increased salinity and transparency occurred during periods of decreased rainfall. The opposite was recorded when rainfall increased, which indicated that the environment was capable of trapping water from continental drainage, even if it was a low quantity, due to the nearness to the channel that links Baía Norte to the Atlantic Ocean. The salinities recorded during the study period were mostly above 30 , which was expected for the study area (MACIEL et al., 2010; SIMONASSI et al., 2010). Nevertheless, the small variation recorded for the salinity is hardly ever found on exposed beaches, where it is rarely a relevant factor for the structure of the ichthyofauna community (GIANNINI; PAIVA FILHO, 1995).

The physicochemical characteristics of Índio beach were significantly correlated to the structure of the fish assemblage. G. barbus and G. genidens had significant negative correlations to transparency, which has also been documented for Ariidae in Baía de Sepetiba (AZEVEDO et al., 1999). The captured biomass was positively correlated to temperature and rainfall, which were higher in spring and summer when they enhanced the productivity in Baía Norte (SIMONASSI et al., 2010) and likely sustained the higher biomasses observed. Higher biomass has also 
been recorded during the hottest months of the year by Santos and Nash (1995) and Suda et al. (2002). On the tidal plains, periods of higher rainfall have been correlated with the increases in fish biomass and richness (SPACH et al., 2004). In addition, prior research has shown that the biomass of demersal fish increased in the middle sector of Baía de Paranaguá during periods of high rainfall (BARLETTA et al., 2008).

The number of individuals caught during the day and/or night was dependent on the sampling month. The meso-scale (months) variations to the assemblage presented an irregular pattern in the number of individuals caught over the course of the months, without a defined trend. However, for shortscale (daytime) variations, the results indicated that on average, the assemblage was more abundant during the day and differed in structure and composition at night. Regular patterns in both the short-scale and meso-scale variations were recorded for the families Ariidae and Tetradontidae, with both of the families having well-defined dynamics of occupation at the beach studied. This has also been ascertained by Ignácio and Spach (2009) for S. testudineus, S. greeleyi, and $G$. genidens on a tidal plain in Baía de Paranaguá.

The number of species found in this study was larger than that recorded on other Brazilian beaches in the states of Paraná, São Paulo, Rio de Janeiro, Espírito Santo, and Bahia (GODEFROID et al., 1997; PESSANHA et al., 2003; FÉLIX et al., 2007; GAELZER; ZALMON, 2008; OLIVEIRASILVA et al., 2008; FÉLIX-HACKDART et al., 2010; VASCONCELLOS et al., 2011). This is, in part, a result of the intermediate physicochemical characteristics found at the site, which ranged between a typical and an estuarine beach, and favored the occurrence of estuarine species at the site (e.g. $S$. testudineus, $S$.greeleyi, Cathorops spixii, Atherinella brasiliensis, Achirus lineatus, Anchoa januaria, Anchoa tricolor, Diapterus rhombeus, Cynoscion microlepidotus, Stellifer stellifer) (GARCIA; VIEIRA, 2001; BARLETTA et al., 2008; REIS-FILHO et al., 2010; VILAR et al., 2011a). However, the low energy of the study site allowed for the use of a capéchade net (visibly efficient in other low-energy environments) for the samplings (IGNÁCIO; SPACH, 2009; VASLET et al., 2010). Given that in most beach studies the sampling is performed using relatively small trawl nets (PESSANHA et al., 2003; GAELZER; ZALMON, 2008; FÉLIX-HACKDART et al., 2010; VASCONCELLOS et al., 2011), which are apparently ineffective at catching individuals that respond rapidly to the visual and mechanical stimulus of the net (GLASS; WARDLE, 1989; METHVEN et al., 2001), the richness of the local ichthyofauna may be underestimated. In addition, nocturnal samplings are essential for evaluating fish diversity because they increase the chances of catching species rarely collected during the day. In the present study, eleven species were recorded exclusively at night (Table 2), which allowed for a better representation of the true community structure (MORRISON et al., 2002). Nevertheless, the number of species recorded was similar to that usually found in shallow environments in southeastern-southern Brazil, which may indicate a pattern of richness in this region.

The species richness caught during the day (77) and at night (74) at Índio beach were very close, with 61 species occurring both during the day and at night. As verified in other shallow areas, the species that were exclusive to a single time of day were not very abundant (GRIFFITHS, 2001; PESSANHA et al., 2003; IGNÁCIO; SPACH, 2009; FÉLIXHACKRADT, 2010; VASCONCELLOS et al., 2011).

The dominance pattern recorded in the present study, in which eight species showed high abundance and relative importance (\%IRI), was similar to various results obtained at other beaches and estuaries worldwide, where generally less than 10 species dominate the environment (SANTOS; NASH, 1995; GIBSON et al., 1993; GRIFFITHS, 2001; STRYDOM, 2003; GODEFROID et al., 1997; PESSANHA et al., 2003; FÉLIX et al., 2007). However, the species that showed high \%IRI in the present study, with the exception of $H$. clupeola, were not classified as dominant species in most of the work carried out on sheltered and exposed beaches from the western portion of the South Atlantic (GODEFROID et al., 1997; PESSANHA et al., 2003; FÉLIXHACKDART et al., 2010; VASCONCELLOS et al., 2011). Sphoeroides testudineus and S. greeleyi are widely distributed in estuarine regions (HACKRADT et al., 2011; VILAR et al., 2011b) and rarely abundant on oceanic beaches (GODEFROID et al., 2003; FÉLIX et al., 2007; GAELZER; ZALMON, 2008; FÉLIX-HACKRADT et al., 2010). A similar pattern has been documented for Genidens genidens and $G$. barbus (MISHIMA; TANJI, 1983; ARAÚJO, 1988; BARLETTA et al., 2008). On the other hand, Harengula clupeola was the most abundant species on sheltered beaches within the states of Paraná (FÉLIXHACKRADT et al., 2010) and Rio de Janeiro (GAELZER; ZALMON, 2008; VASCONCELLOS et al., 2011), and Cetengraulis edentulus and Anchoviella lepidentostole were apparently abundant in the coastal regions of the state of Santa Catarina (BARREIROS et al., 2004; SOUZA-CONCEIÇÃO, 2005). The demersal species that composed a large portion of the $\%$ IRI in the present study were also abundant in demersal trawls in Baía Sul (Florianópolis) (CARTAGENA et al., 2011). Parameters such as spatial differences (as mentioned in the studies cited) and different selectivity among sampling gear can 
affect abundance and/or dominance (ROZAS; MINEILO, 1997). The most important species in this study were selected based on the \%IRI, which considered the proportional contribution of each variable (weight, $\mathrm{FO} \%$ and abundance) in the index. Thus, a species with low weight (g) and low FO\%, regardless of its abundance, may show smaller \%IRI values than species with higher weight and $\mathrm{FO} \%$, as observed for $H$. clupeola and $S$. testudineus in this study.

At Índio beach, abundance had a tendency to increase in the hottest months, i.e. November to April, a finding which is congruent with those of other studies. In the Gulf of Mexico (ROSS et al., 1987) and Scotland (GIBSON, 1973) ichthyofauna abundance peaks were recorded at the end of spring and in summer. In addition, on beaches of the Azores archipelago (SANTOS; NASH, 1995) and in Japan (SUDA et al., 2002), higher abundance and biomass were recorded in the hottest months of the year. Higher abundance was also found during the summer by Bennett (1989) in South Africa, unlike that observed by Lasiak (1984b) and Clark et al. (1996) in the same region. The rise in the average abundance during the hottest periods of the year seems to result from, first, the spawning peaks of some species (e.g. $S$. rastrifer and $C$. edentulus) and, second, the recruitment peaks of other species (e.g. G. barbus and S. testudineus).

The short-scale biomass variations (day and night) were not significant at Índio Beach; however, the biomass was slightly higher in the nocturnal catches, which may indicate a possible entry process of larger individuals at night (NASH et al., 1994). Statistically, for the species with higher \%IRI, only larger-sized individuals of $C$. edentulus were documented at night. On the other hand, the specific composition of the average biomass for each time of day varied widely and was molded by the composition of the ichthyofauna in each period, as observed by MORRISON et al. (2002).

Of the species with higher \%IRI, only $S$. greeleyi and $A$. lepidentostole had an interaction (ANOVA) in the abundance between the time of day and the month of sampling. Vasconcellos et al. (2011) found an interaction between the season and the time of day for Atherinella brasiliensis, Pomatomus saltatrix, Trachinotus carolinus, Umbrina coroides and Anchoa lyoleps, which showed an inconsistent pattern of nycthemeral occupation within the beach environment. Higher nocturnal richness was also found by Methven et al. (2001) in a shallow area of the estuary in Newfoundland, with two groups of species - one showing predominance at night and another during the day. On tidal plains in a temperate estuary in New Zealand, the ichthyofauna presented higher richness and abundance at night, with interaction (ANOVA) between the tide and the time of day for two of the eight most abundant species (MORRISON et al., 2002). The small tidal amplitude $(\sim 0.52 \mathrm{~m})$ at Índio beach (SORIANO-SIERRA; SIERRA DE LEDO, 1998), and the standardization of the sampling at neap tide in the present study decreased the tidal effect on the catches. However, even though this effect was reduced, it may have influenced the catches, given that the dynamics of the ichthyofauna varied widely between the tidal regimes, with a possible concentration of fish in channels at low tide and an exploitation of the floodplains at high tide (GIBSON et al., 1996; MORRISON et al., 2002; OLIVEIRA-NETO et al., 2008).

To understand the aspects that govern the movement of specific groups to greater depths, trophic analyses between the sampled ichthyofauna and the beach fauna, could be promoted (NAGELKERKEN et al., 2000; SOARES; VAZZOLER, 2001). A greater number of ariids were collected at night at Índio beach and were also associated with lower depths at night in Baía Sul (Florianópolis) (CARTAGENA et al., 2011). These fish use barbells to help them recognize food items on the bottom (CHAVES; VENDEL, 1996), which is a morphological characteristic that may favor the nocturnal activity of the family (also recorded for Polydactylus virginicus). During the day, the ariids seem to be associated with deeper environments $(>3 \mathrm{~m})$ (SCHWARZ Jr. et al., 2007; CARTAGENA et al., 2011), which is an adaptation that most likely decreases the interspecific competition and predation risk from birds in shallow areas (HYNDES et al., 1996; YOUNG et al., 1997; CLARK et al., 2003).

Another explanation for the nycthemeral variation in the abundance of the ariids could be physiological. Among the species with higher \%IRI, only $G$. barbus and $G$. genidens presented negative correlations with transparency, in addition to being preferentially nocturnal. Some fish species are particularly deprived, or possess low quantities of skin compounds called photoprotectors (FABACHER; LITTLE, 1995). These compounds are mainly melanin pigments capable of absorbing UV radiation. Melanin may vary in quantity according to the species and the stage of life (FABACHER; LITTLE, 1995; HADER et al., 2007); however, its production can also be stimulated. Individuals exposed to high levels of radiation at the surface present high concentrations of melanin (ADACHI et al., 2005), which may lead to a consequent energy expenditure. In this context, the levels of solar radiation, which vary according to the depth, the water transparency and the time of day, could be influencing the displacement of the ariids.

The high number of individuals of $S$. testudineus and $S$. greeleyi recorded during daytime samplings coincided with their feeding activity on crustaceans (Brachyura and Penaeidae) and mollusks 
(Gastropoda and Bivalvia) (SANTOS; RODRIGUEZ, 2011).

The results obtained at Índio beach for the other families and species also showed short-scale variations; however, in most cases, the small number of specimens collected or the occurrence of seasonal peaks, guided the formulations of hypotheses and cautious inferences. Gerreidae were more abundant during the day, while Haemulidae were more abundant at night. These findings were similar to those found in a Caribbean estuary where Gerreidae were more abundant during the day in mangrove environments, and Haemulidae were more abundant at night in algal banks (NAGELKERKEN et al., 2000). In this study, Sciaenidae presented high species abundance and richness, with a tendency for higher nocturnal occurrences, a finding that was also verified by Ignácio and Spach (2009) and Barreiros et al. (2005). On the South-southeastern continental shelf, members of Sciaenidae feed during the entire daytime period, with some species showing preference for nighttime or daytime food resources, indicating a possible resource partitioning strategy for coexistence (SOARES; VAZZOLER, 2001). Anchoa januaria was present in the winter and spring, with higher abundance during the day. Anchoa januaria feeds on zooplankton during the day and exploits different strata of the water column. In winter, when there is lower availability of planktonic food, this small pelagic species mainly eats Harpacticoida copepods from interstitial fauna (SERGIPENSE et al., 1999), which is probably associated with the catches of these species in a beach environment. The daytime predominance of $C$. spilopteturs was most likely a result of its consumption of mysids and polychaetes (TOEPFER; FLEEGER, 1995), which was facilitated during the day in shallow areas by the morphological characteristics of this visual predator (GUEDES, op. cit.).

Fish that are sensitive to changes in the sunlight during the crepuscular periods of dawn and dusk can respond physiologically (SPIELER; NOESKE, 1981) or ecologically (HYNDES et al., 1996; YOUNG et al., 1997; CLARK et al., 2003), or may gradually change their activity within beach environments. Thus the pattern of nycthemeral variation verified for several species could have been more prominent if a crepuscular interval (i.e. 2 hours) were added between the diurnal and nocturnal samplings. Moreover, measuring the level of nocturnal luminosity might be a way to determine if the monthly and day/night abundance interactions that were calculated using the ANOVAs as well as the elevation in the nocturnal averages for predominantly diurnal species could be verified.

\section{REFERENCES}

ADACHI, K.; KATO, K.; WAKAMATSU, K.; ITO, S. ISHIMARU, K.; HIRATA, T.; MURATA, O.; KUMAI, $\mathrm{H}$. Histological analysis, colorimetric evaluation, and chemical quantification of melanin content in 'suntanned' fish. Pigm. Cell Res., v. 18, n. 6, p. 465468, 2005.

ARAÚJO, F. G. Distribuição, abundância relativa e movimentos sazonais de bagres marinhos (Siluriformes, Ariidae) no estuário da Lagoa dos Patos (RS), Brasil. Rev. Bras. Zool., v. 5, n. 4, p. 509-543, 1988.

ARAUJO, C. C. V. D.; ROSA, D. D. M.; FERNANDES, J. M.; RIPOLI, L. V.; KROHLING, W. Composição e estrutura da comunidade de peixes de uma praia arenosa da Ilha do Frade, Vitória, Espírito Santo. Iheringia, Ser. Zool., v. 98, n. 1, p. 129-135, 2008.

AZEVEDO, M. C. C.; ARAÚJO, F. G.; CRUZ-FILHO, A. G.; GOMES, I. D.; PESSANHA, A. L. M. Variação espacial e temporal de bagres marinhos (Siluriformes, Ariidae) na Baía de Sepetiba, Rio de Janeiro. Rev. Bras. Biol., v. 59, n. 3, p. 443-454, 1999.

BARLETTA, M.; AMARAL, C. S.; CORRÊA, M. F. M.; GUEBERT, F.; DANTAS, D. V.; LORENZI, L.; SAINT-PAUL, U. Factors affecting seasonal variations in demersal fish assemblages at an ecocline in a tropicalsubtropical estuary. J. Fish Biol., v. 73, n. 6, p. 13141336, 2008

BARLETTA, M.; CORRÊA, M. F. M. Guia para identificação de peixes da costa do Brasil. Curitiba: UFPR, 1992. $131 \mathrm{p}$.

BARREIROS, J. P.; FIGNA, V.; HOSTIM-SILVA, M.; SANTOS, R. S. Seasonal changes in a sandy beach fish assemblage at Canto Grande, Santa Catarina, South Brazil. J. Coastal Res., v. 20, n. 3, p. 862-870, 2004.

BARREIROS, J. P.; FIGNA, V.; HOSTIM-SILVA, M.; SANTOS, R. S. Diel seasonality of a shallow-water fish assemblage at Canto Grande, Santa Catarina, Brazil. J. Coastal Res., v. 42, p. 343-347, 2005.

BASTOS, G. C.; PETRERE Jr., M. Small-scale marine fisheries in the municipal district of Florianópolis, Santa Catarina, Brazil. Braz. J. Biol., v. 70, n. 4, p. 947-953, 2010.

BENNETT, B. A. The fish community of a moderately exposed beach on the Southwestern Cape cost of South Africa and assessment of this habitat as a Nursey for juvenile fish. Estuarine, Coastal Shelf Sci., v. 28, n. 3, p. 293-305, 1989.

BORZONE, C. A.; MELO, S. G.; REZENDE, K. V.; DO VALE, R.; KRUL, R. Macrobenthic intertidal communities from wave to tide dominated beach environments: a case study in two Brazilian beaches. J. Coastal Res., v. 35, sp. iss., p. 472-480, 2003.

CARTAGENA, B. F. C.; HOSTIM-SILVA, M.; SPACH, H. L. Distribuição espacial da ictiofauna demersal no Saco dos Limões (Baía Sul, Florianópolis, SC). Bioikos, v. 25, n. 2, p. 117-128, 2011.

CHAVES, P. T. C.; VENDEL, A. L. Aspectos da alimentação de Genidens genidens (Valenciennes) (Siluriformes, Ariidae) na Baía de Guaratuba, Paraná. Rev. Bras. Zool., v. 13, n. 3, p. 669-675, 1996. 
CLARK, B. M.; BENNET, B. A.; LAMBERTH, S. J. Factors affecting spatial variability in seine net catches of fishes in the surf zone of False Bay, South Africa. Mar. Ecol.: Prog. Ser., v. 131, p. 17-34, 1996.

CLARK, K. L.; RUIZ, G. M.; HINES, A. H. Diel variation in predator abundance, predation risk and prey distribution in shallow-water estuarine habitats. J. Exp. Mar. Biol. Ecol., v. 287, n. 1, p. 37-55, 2003.

CLARKE, K. R. Non-parametric multivariate analyses of changes in community structure. Aust. J. Ecol., v. 18, n. 1, p. 117-143, 1993.

CLARKE, K. R.; WARWICK, R. M. Change in marine communities: an approach to statistical analysis and interpretation. Plymouth: Natural Environment Research Council, 1994. 144 p.

CLARKE, K. R.; GORLEY, R. N. PRIMER v6: user manual/tutorial. Plymouth: PRIMER-E, 2006. $192 \mathrm{p}$.

COSTA, M. D. P.; SOUZA-CONCEIÇÃO, J. M. Composição e abundância de ovos e larvas de peixes na baía da Babitonga, Santa Catarina, Brasil. Pan-Am. J. Aquat. Sci., v. 4, n. 3, p. 372-382, 2009.

FABACHER, D. L.; LITTLE, E. E. Skin component may protect fishes from ultraviolet-B radiation. Environ. Sci. Pollut. Res., v. 2, n. 1, p. 30-32, 1995.

FAO. World review of fisheries and aquaculture: part 1 . 2010. 89 p. Available in: http://www.fao.org/docrep/013/i1820e/i1820e01.pdf. Access on: 30th May 2012.

FÉLIX, F. C.; SPACH, H. L.; MORO, P. S.; SCHWARZ Jr., R.; SANTOS, C.; HACKRADT, C. W.; HOSTIMSILVA, M. Utilization patterns of surf zone inhabiting fish from beaches in Southern Brazil. Pan-Am. J. Aquat. Sci., v. 2, n. 1, p. 27-39, 2007.

FÉLIX-HACKRADT, F. C.; SPACH, H. L.; MORO, P. S.; PICHLER, H. A.; MAGGI, A. S.; HOSTIM-SILVA, M.; HACKRADT, C. W. Diel and tidal variation in surf zone fish assemblages of a sheltered beach in Southern Brazil. Lat. Am. J. Aquat. Res., v. 38, n. 3, p. 447-460, 2010.

FIGUEIREDO, J. L.; MENEZES, N. A. Manual de peixes marinhos do Sudeste do Brasil. II. Teleostei (1). São Paulo: Museu de Zoologia da USP, 1978. 110 p.

FIGUEIREDO, J. L.; MENEZES, N. A. Manual de peixes marinhos do sudeste do Brasil. III. Teleostei (2). São Paulo: Museu de Zoologia da USP, 1980. 90 p.

FIGUEIREDO, J. L.; MENEZES, N. A. Manual de peixes marinhos do sudeste do Brasil. VI. Teleostei (5). São Paulo: Museu de Zoologia da USP, 2000. 116 p.

GAELZER, L. R.; ZALMON, I. R. Diel variation of fish community in sandy beaches of Southeastern Brazil. Braz. J. Oceanogr., v. 56, n. 1, p. 23-39, 2008.

ROSENTAL ZALMON, I.; GAELZER, L. R. The influence of wave gradient on the ichthyofauna of Southeastern Brazil: focusing the community structure in surf zone. J. Coastal Res., v. 35, sp. iss., p. 456-462, 2003.

GARCIA, A. M.; VIEIRA, J. P. O aumento da diversidade de peixes no estuário da Lagoa dos Patos durante o episódio El Niño 1997-1998. Atlântica, v. 23, p. 85-96, 2001.

GIANNINI, R.; PAIVA-FILHO, A. M. Análise comparativa da ictiofauna da zona de arrebentação de praias arenosas do Estado de São Paulo, Brasil. Bol. Inst. Oceanogr., v. 43, n. 2, p. 141-152, 1995.

GIBSON, R. N. The intertidal movements and distribution of young fish on a sandy beach with special reference to the plaice (Pleuronectes platessa L.). J. Exp. Mar. Biol. Ecol., v. 12, n. 1, p. 79-102, 1973.

GIBSON, R. N.; ANSELL, A. D.; ROBB, L. Seasonal and annual variations in abundance and species composition of fish and macrocrustacean communities on a Scottish sandy beach. Mar. Ecol.: Prog. Ser., v. 98, n. 1/2, p. 117, 1993.

GIBSON, R. N.; ROBB, L.; BURROWS, M. T.; ANSELL, A. D. Tidal, diel and longer term changes in the distribution of fishes on a Scottish sandy beach. Mar. Ecol.: Prog. Ser., v. 130, p. 1-17. 1996.

GLASS, C. W.; WARDLE, C. S. Comparison of the reactions of fish to a trawl gear, at high and low light intensities. Fish. Res., v. 7, n. 3, p. 249-266, 1989.

GODEFROID, R. S.; HOFSTAETTER, M.; SPACH, H. L. Structure of the fish assemblage in the surf zone of the beach at Pontal do Sul, Paraná. Nerítica, v. 11, p. 77-93, 1997.

GODEFROID, R. S.; SPACH, H. L.; SCHWARZ Jr., R.; QUEIROZ, G. M. L. N. A fauna de peixes da praia do Balneário Atami, Paraná, Brasil. Atlântica, v. 25, n. 2, p. 147-161, 2003.

GRIFFITHS, S. P. Diel variation in the seagrass ichthyofaunas of three intermittently open estuaries in South-eastern Australia: implications for improving fish diversity assessments. Fish. Manage. Ecol., v.8, n. 2, p. 123-140. 2001.

GUEDES, A. P. P.; ARAÚJO, F. G.; AZAVEDO, M. C. C. Estratégia trófica dos linguados Citharichthys spilopterus Günther e Symphurus tesselatus (Quoy \& Gaimard) (Actinopterygii, Pleuronectiformes) na Baía de Sepetiba, Rio de Janeiro. Rev. Bras. Zool., v. 21, n. 4, p. 857-864, 2004.

HACKRADT, C. W.; FÉLIX-HACKRADT, F. C.; PICHLER, H. A.; SPACH, H. L.; SANTOS, L. O. Factors influencing spatial patterns of the ichthyofauna of low energy estuarine beaches in Southern Brazil. J. Mar. Biol. Assoc. U. K., v. 91, sp. iss. 6, p. 1345-1357, 2011.

HADER, D. P.; KUMAR, H. D.; SMITH, R. C.; WORREST, R. C. Effects of solar UV radiation on aquatic ecosystem and interactions with climate change. Photochem. Photobiol. Sci., v. 6, p. 167-285, 2007.

HOEFEL, F. G. Morfodinâmica de praias arenosas ocêanicas: uma revisão bibliográfica. Itajaí: Universidde do Vale do Itajaí, 1998. 92 p.

HYNDES, G. A.; POTTER, I. C.; LENANTON, R. C. J. Habitat partitioning by whiting species (Sillaginidae) in coastal waters. Environ. Biol. Fishes, v. 45, n. 1, p. 21 40, 1996.

IBGE. Censo 2010: resultados gerais da amostra. 2010. Disponível em: http://www.censo2010.ibge.gov.br/amostra/. Access on: 21th May 2012.

IGNÁCIO, J. M.; SPACH, H. L. Variação entre o dia e a noite nas características da ictiofauna do infra-litoral raso do Maciel, Baía de Paranaguá, PR. Rev. Bras. Zoocienc., v. 11, n. 1, p. 25-37, 2009.

JACKSON, N. L.; NORDSTROM, K. F.; ELIOT, I.; MASSELINK, G. 'Low energy' sandy beaches in marine and estuarine environments: a review. Geomorphology, v. 48 , n. $1 / 3$, p. 147-162, 2002. [29th Binghamton Geomorphology Symposium: Coastal Geomorphology]. 
KENNISH, M. J. Environmental threats and environmental future of estuaries. Environ. Conserv., v. 29, n. 1, p. 78107, 2002.

LASIAK, T. A. Structural aspects of the surf zone fish assemblage at King's Beach, Algoa Bay, South Africa: short-term fluctuations. Estuarine, Coastal Shelf Sci., v. 18, n. 3, p. 347-360, 1984a.

LASIAK, T. A. Structural aspects of the surf zone fish assemblage at King's Beach, Algoa Bay, South Africa: long-term fluctuations. Estuarine, Coastal Shelf Sci., v. 18 , n. 4, p. 459-483, 1984b.

LIN, H. J.; SHAO, K. T. Seasonal and diel changes in a subtropical mangrove fish assemblage. Bull. Mar. Sci., v. 65, n. 3, p. 775-794, 1999.

LOUIS, M.; BOUCHON, C.; BOUCHON-NAVARO, Y. Spatial and temporal variations of mangrove fish assemblages in Martinique (French West Indies). Hydrobiologia, v. 295, n. 1/3, p. 275-284, 1995.

MACIEL, M. L. T.; IBBOTSON, D. P.; MAGALHÃES, A. R. M. Polidiariose em ostras Crassostrea gigas cultivadas na Praia da Ponta do Sambaqui, Florianópolis, Santa Catarina - Brasil. Braz. J. Vet. Res. Animal Sci., v. 47, n. 5, p. 337-345, 2010.

MARCENIUK, A. P. Chave de identificação das espécies de bagres marinhos (Siluriformes, Ariidae) da costa brasileira. Bol. Inst. Pesca, v. 31, n. 2, p. 89-101, 2005.

MARTINS, R. S.; PEREZ J. A. A. Artisanal fish-trap fishery around Santa Catarina Island during spring/summer?: characteristics, species interactions and the influence of the winds on the catches. Bol. Inst. Pesca, v. 34, n.3, p. 413-423, 2008

MCLACHLAN, A.; DORVLO, A. Global patterns in sandy beach macrobenthic communities. J. Coastal Res., v. 21, n. 4, p. 674-687, 2005

MENEZES, N. A.; FIGUEIREDO, J. L. Manual de peixes marinhos do sudeste do Brasil. IV. Teleostei (3). São Paulo: Museu de Zoologia da USP, 1980. 96 p.

MENEZES, N. A.; FIGUEIREDO, J. L. Manual de peixes marinhos do sudeste do Brasil. V. Teleostei (4). São Paulo: Museu de Zoologia da USP, 1985. 105 p.

METHVEN, D. A.; HAEDRICH, R. L.; ROSE, G. A. The fish assemblage of a Newfoundland Estuary: diel, monthly and annual variation. Estuarine, Coastal Shelf Sci., v. 52, n. 6, p. 669-687. 2001.

MISHIMA, M.; TANJI, S. Fatores ambientais relacionados à distribuição e abundância de bagres marinhos (Osteichthys, Ariidae) no complexo estuarino lagunar de Cananéia $\left(25^{\circ} \mathrm{S}, 48^{\circ} \mathrm{W}\right)$. Bol. Inst. Pesca, v. 10 , p. 17-27, 1983.

MORRISON, M. A.; FRANCIS, M. P.; HARTILL, B. W.; PARKINSON, D. M. Diurnal and tidal variation in the abundance of the fish fauna of a temperate tidal mudflat. Estuarine, Coastal Shelf Sci., v. 54, n. 5, p. 793-807, 2002.

NAGELKERKEN, I.; DORENBOSCH, M.; VERBERK, W. C. E. P.; COCHERET DE LA MORINIÈRE, E.; VAN DER VELDE, G. Day-night shifts of fishes between shallow-water biotopes of a Caribbean bay, with emphasis on the nocturnal feeding of Haemulidae and Lutjanidae. Mar. Ecol.: Prog. Ser., v. 194, p. 55-64, 2000.

NASH, R. D. M.; SANTOS, R. S.; GEFFEN, A. J.; HUGHES, G.; ELLIS, T. R. Diel variability in catch rate of juvenile flatfish on two small nursery grounds (Port
Erin Bay, Isle of Man and Porto Pirn Bay, Faial, Azores). J. Fish Biol., v. 44, n. 1, p. 35-45, 1994.

OLIVEIRA-SILVA, J. T.; PESO-AGUIAR, M. C.; LOPES, P. R. D. Ictiofauna das praias de Cabuçu e Berlinque: uma contribuição ao conhecimento das comunidades de peixes na Baía de Todos os Santos - Bahia - Brasil. Biotemas, v. 21, n. 4, p. 105-115, 2008.

OLIVEIRA-NETO, J. F.; SPACH, H. L.; SCHWARZ-Jr., R.; PICHLER, H. A. Diel variation in fish assemblages in tidal creeks in Southern Brazil. Braz. J. Biol., v. 68, n. 1, p. 37-43, 2008.

PAGLIOSA, P. R.; BARBOSA, F. A. R. Assessing the environment-benthic fauna coupling in protected and urban areas of Southern Brazil. Biol. Conserv., v. 129, n. 3, p. 408-417, 2006.

PESSANHA, A. L. M.; ARAÚJO, F. G.; AZEVEDO, M. C. C.; GOMES, I. D. Diel and seasonal changes in the distribution of fish on a Southeast Brazil sandy beach. Mar. Biol., v. 143, n. 6, p. 1047-1055, 2003.

PINKAS, L.; OLIPHANT, M. S.; IVERSON, I. L. K. Food habits of Albacore, Bluefin Tuna, and Bonito in California waters. 1971. p. 1-105. (California Department of Fish and Game Fish Bulletin, v. 152). Available

in: http://content.cdlib.org/view?docId=kt8290062w\&brand $=$ calisphere\&doc.view=entire_text. Access on: dia mês abrev. ano.

REIS-FILHO, J. A.; NUNES, J. A. C. C.; FERREIRA, A. Estuarine ichthyofauna of the Paraguaçu River, Todos os Santos Bay, Bahia, Brazil. Biota Neotrop., v. 10, n. 4, p. 301-312, 2010.

RIBEIRO, G. C.; CLEZAR, L.; SILVA, M. H. Abundância e distribuição espaço-temporal dos Gerreidae (Pisces) na Lagoa da Conceição e área costeira adjacente, Ilha de Santa Catarina. Aquitaine Ocean, v. 3, p. 281-286, 1997.

RIBEIRO, G. C.; CLEZAR, L.; SILVA, M. H. Comunidade ictíica, sua variação espacial e sazonal na Lagoa da Conceição e área costeira, Ilha de Santa Catarina, SC, Brasil: o ecossistema da Lagoa da Conceição. Ser. Fepema, v. 4, p. 261-273, 1999.

ROSA, L. C.; BORZONE, C. A. Uma abordagem morfodinâmica na caracterização física das praias estuarinas da Baia de Paranguá, Sul do Brasil. Rev. Bras. Geocienc., v. 38, n. 2, p. 237-245. 2008.

ROSS, S. T.; MCMICHAEL Jr., R. H.; RUPLE, D. L. Seasonal and diel variation in the standing crop of fishes and macroinvertebrates from a Gulf of Mexico surf zone. Estuarine, Coastal Shelf Sci., v. 25, n. 4, p. 391-412, 1987.

ROZAS, L. P.; MINELLO, T. J. Estimating densities of small fishes and decapod crustaceans in shallow estuarine habitats: a review of sampling design with focus on gear selection. Estuaries, v. 20, n. 1, p. 199213, 1997.

RUPLE, D. L. Occurrence of larval fishes in the surf zone of a Northern Gulf of Mexico barrier island. Estuarine, Coastal Shelf Sci., v. 18, n. 2, 191-208, 1984.

SANTOS, R. S.; NASH, R. D. M. Seasonal changes in a sandy beach fish assemblage at Porto Pim, Faial, Azores. Estuarine, Coastal Shelf Sci., v. 41, n. 5, p. 579-591, 1995. 
SANTOS, A. C. A.; RODRIGUEZ, F. N. C. Ocorrência e alimentação do Baiacu Sphoe

roides testudineus (Actinopterygii - Tetraodontiformes) na margem Oeste da Baía de Todos os Santos, Bahia, Brasil. Sitientibus: Ser. Cienc. Biol., v. 11, n. 1, p. 3136, 2011.

SCHWARZ Jr., R.; FRANCO, A. C. N. P.; SPACH, H. L.; SANTOS, C.; PICHLER, H. A.; QUEIROZ, G. M. L. N. Variação da estrutura espacial da ictiofauna dermensal capturada com rede de arrasto de porta na Baía dos Pineiros, PR. Bol. Inst. Pesca, v. 33, n. 2, p. 157-169, 2007.

SERGIPENSE, S.; CARAMASCHI, E. P.; SAZIMA, I. Morfologia e hábitos alimentares de duas espécies de Engraulidae (Teleostei-Clupeiformes) na Baía de Sepetiba, Rio de Janeiro. Rev. Bras. Oceanogr., v. 47, n. 2, p. 173-188, 1999.

SILVEIRA, L. F.; KLEIN, A. H. F.; TESSLER, M. G. Classificação morfodinâmica das praias do estado de Santa Catarina e do litoral Norte do Estado de São Paulo utilizando sensoriamento remoto. Braz. J. Aquat. Sci. Technol., v. 15, n. 2, p. 13-28, 2011.

SIMONASSI, J. C.; HENNEMANN, M. C. TALGATTI, D.; MARQUES Jr., A. N. Nutrient variations and coastal water quality of Santa Catarina Island, Brazil. Biotemas, v. 23, n. 1, p. 211-223, 2010.

SOARES, L. S. H.; VAZZOLER, A. E. A. M. Diel changes in food and feeding activity of sciaenid fishes from the South-western Atlantic, Brazil. Rev. Bras. Biol., v. 61, n. 2, p. 197-216, 2001.

SORIANO-SIERRA, E.; SIERRA DE LEDO, B. (Eds.). Ecologia e gerenciamento do manguezal de Itacorubí. Florianópolis: NEMAR: CCB: UFSC, 1998. 396 p.

SOUZA-CONCEIÇÃO, J. M.; RODRIGUES-RIBEIRO, M.; CASTRO-SILVA, M. A. Dinâmica populacional, biologia reprodutiva e o ictioplâncton de Cetengraulis edentulus Cuvier (Pisces, Clupeiformes, Engraulidae) na enseada do Saco dos Limões, Florianópolis, Santa Catarina, Brasil. Rev. Bras. Zool., v. 22, n. 4, p. 953961, 2005.

SPACH, H. L.; GODEFROID, R. S.; SANTOS, C.; SCHWARZ Jr., R.; QUEIROZ, G. M. L. Temporal variation in fish assemblage composition on a tidal flat. Braz. J. Oceanogr., v. 52, n. 1, p. 47-58, 2004.

SPIELER, R. E.; NOESKE, T. A. Timing of a single daily meal and diel variations of serum thyroxine, triiodothyronine and cortisol in goldfish Carassius auratus. Life Sci., v. 28, n. 26, p. 2939-2944, 1981.

STRYDOM, N. A. Occurrence of larval and early juvenile fishes in the surf zone adjacent to two intermittently open estuaries, South Africa. Environ. Biol. Fishes, v. 66, n. 4, p. 349-359, 2003.
SUDA, Y.; INOUE, T.; UCHIDA, H. Fish communities in the Surf Zone of a protected sandy beach at Doigahama, Yamaguchi Prefecture, Japan. Estuarine, Coastal Shelf Sci., v. 55, n. 1, p. 81-96, 2002.

SUGUIO, K. Introdução à sedimentologia. São Paulo: Edgard Blücher: EDUSP, 1973. 371 p.

TOEPFER, C. S.; FLEEGER, W. Diet of juvenile fishes Citharichthys spilopterus, Symphurus plagiusa, and Gobionellus boleosoma. Bull. Mar. Sci., v. 56, n. 1, p. 238-249, 1995.

UNDERWOOD, A. J. Experiments in Ecology: their logical design and interpretation using analysis of variance. Cambridge: New York: Cambridge University Press. 1997. 504 p.

VASCONCELLOS, R. M.; ARAÚJO, F. G.; SANTOS, J. N. S.; SILVA, M. A. Diel seasonality in fish biodiversity in a sandy beach in South-eastern Brazil. J. Mar. Biol. Assoc. U. K., v. 91, sp. iss. 6, p. 1337-1344, 2011.

VASLET, A.; BOUCHON-NAVARO, Y.; CHARRIER, G.; LOUIS, M.; BOUCHON, C. Spatial patterns of mangrove shoreline fish communities in relation with (to) environmental variables in Caribbean Lagoons. Estuaries Coasts, v. 33, n. 1, p. 195-210, 2010.

VILAR, C. C.; SPACH, H. L.; OLIVEIRA SANTOS, L. Fish fauna of Baía da Babitonga (Southern Brazil), with remarks on species abundance, ontogenic stage and conservation status. Zootaxa, v. 2734, p. 40-52, 2011 a.

VILAR, C. C.; SPACH, H. L.; SOUZA-CONCEIÇÃO, J. M. Fish assemblage in shallow areas of Baía da Babitonga, Southern Brazil: structure, spatial and temporal patterns. Pan-Am. J. Aquat. Sci., v. 6, n. 4, p. 303-319, 2011 b.

WRIGHT, J. M. Diel variation and seasonal consistency in the fish assemblage of the non-estuarine Sulaibikhat Bay, Kuwait. Mar. Biol., v. 102, n. 1, p. 135-142, 1989.

YOUNG, G. C.; POTTER, I. C.; HYNDES, G. A.; LESTANG, S. The ichthyofauna of an intermittently open estuary: Implications of bar breaching and low salinities on faunal composition. Estuarine, Coastal Shelf Sci., v. 45, n. 1, p. 53-68, 1997.

(Manuscript received 17 June 2013; revised 21 January 2014; accepted 19 May 2014) 